\title{
Comments for going with the flow the promise and challenge of liquid biopsies.
}

\section{Jia-Qing Huang, Zhong Jia*}

Department of Hepatobiliary Surgery, Affiliated Hangzhou First People's Hospital, Zhejiang University School of Medicine \& Zhejiang Chinese Medical University Affiliated Hangzhou First Hospital, Hangzhou 310006, PR China

Keywords: Liquid biopsies, Translational medicine, Tumors.

Accepted on April 10, 2018

\section{To the Editor:}

We go with greatly interest through the article by Friedrich [1] which describe a hot topic concerning on liquid biopsies. It appears to be enthralled since liquid biopsies such as blood sample by detectable CTCs and/or ctDNA results from tumor releasing or oncolytic form through an unknown mechanism, just like a patent extra-tumor "drone". The bottleneck the author describe-promises powerfully associated with challenges-appears rather typical for oncologists and researchers in fields of translational medicine and therefore does add much information to the potential readers of a specialized issue despite update no any encouraging breakthrough has been obtained. Despite CTC or ctDNA plays an important role via liquied biopsies, still, the non-invasive testing for pancreatic cystic disease may be a difficult point as cystic liquied biopsy remains great contraversial in real world. Also, we note that shrinking or restricting these "nude" tumor cells (ctDNA) and "free" ones (CTCs) not detecting them alone may be more valuable.
In summary, liquied biopsies, including CTC, ctDNA, tumor markers, etc. need more practices to evaluate its true practical significance and implications.

\section{References}

1. Friedrich MJ. Going with the flow: the promise and challenge of liquid biopsies. JAMA 2017; 318: 1095-1097.

\section{*Correspondence to}

Zhong Jia

Department of Hepatobiliary Surgery

Affiliated Hangzhou First People's Hospital

Zhejiang University School of Medicine

PR China 